Herr Dr. R. Marc-Jena:

\title{
ÜBER DIE KRISTALLISATION AUS WÄSSERIGEN LÖSUNGEN.
}

Der große Fortschritt, den unsere Kenntnis über den Mechanismus homogener Reaktionen durch die Kinetik gemacht hat, ließ es sehr erstrebenswert erscheinen, auch die Kinetik der Vorgänge in heterogenen Systemen zu studieren. In jüngster Zeit haben die Arbeiten von Bodenstein und seinen Schülern fär eine Reihe chemischer Vobrgange in heterogenen Systemen aufklärende Resultate gezeitigt, doch wäre es natürlich verfrüht, ihre Befunde verallgemeinernd auf sämtliche heterogenen Vorgänge ausdehnen zu wollen. Zumal Vorgänge, wie die der Auflösung und der Kristallisation scheinen uns eine gewisse Sonderstellung einzunehmen. Für diese schienen die Theorien von Noyes und Whitney sowie Nernst und Brunner eine hinreichende Erklärung zu geben, und es bestand daher relativ wenig Hoffnung, auf Grund kinetischer Studien etwas über den Chemismus der Kristallisation zu erfahren. Eine Reihe verschiedener Beobachtungen brachte mich jedoch $\mathrm{zu}$ der Ueberzeugung, daß für den Vorgang der Kristallisation das Nernst-Noyessche Gesetz strenge Gültigkeit nicht beanspruchen könne, d. h., daß die Geschwindigkeit des Vorganges an der Grenzfläche nicht als unmeßbar groß angesehen werden dürfe.

Ich habe nun bereits vor etwa $I / / 2$ Jahren mitteilen können, daß es gelingt, die Kristallisation des Kaliumsulfats unter gewissen $\mathrm{Be}$ dingungen so zu leiten, daß die Kristallisationsgeschwindigkeit von der Rürgeschwindigkeit unabhăngig wird. Dies würde beweisen, da $B$, wenn eine Diffusionsschicht tatsächlich am Kristall vorhanden ist, die die Konzentration der Sättigung hat, diese oberhalb einer gewissen Rührgeschwindigkeit unveränderlich wird, also als durch gewisse Kräfte mit dem Kristall starr verbunden $z u$ betrachten ware. Gegen einen Diffusionsvorgang überhaupt sprach aber die andere von mir gefundene Tatsache, daß nämlich die Geschwindigkeit des Vorganges sehr genau dem Quadrat der Uebersăttigung proportional war.

Nun wäre es allerdings möglich, diese Tatsache dadurch zu erklären, daß sich einzelne Vorgänge in irgendwelcher Weise superponierten, zumal meine Methode, die darin besteht, eine gewisse Menge gut ausgebildeten, grobkörnigen Kristallpulvers (etwa $0,2 \mathrm{~mm}$-Kristalle) in der Lösung mit etwa Iooo Touren Geschwindigkeit zu wirbeln, nicht ohne weiteres ganz einwandfrei erscheinen könnte und die Möglichkeit einer undefinierten Aenderung der Oberfläche nicht von der Hand zu weisen ist. Um diesen Einwänden zu begegnen, habe ich zwei Wege eingeschlagen.
Erstens habe ich eine größere Reihe von Stoffen untersucht, und zweitens habe ich gezeigt, daß sich die Oberfläche der Kristallkeime in genau berechenbarer Weise ändert.

Die Auswahl der Stoffe ist ziemlich schwierig; sie dürfen nicht zu leicht löslich sein, weil sonst die Uebersattigung in einem ungunstigen Verhältnis zur Gesamtkonzentration steht, und sie müssen sich gut übersättigen lassen. Schließlich war es wünschenswert, Stoffe mit und ohne Kristallwasser und mit verschiedenen Säureradikalen zu untersuchen. Es wurden definitiv gemessen: mittellösliche: Ammoniak- und Kalialaun, Kaliumsulfat, Kaliumbichromat; schwerlösliche: Silberacetat.

Alle diese fünf Stoffe kristallisierten mit einer Geschwindigkeit, die dem Quadrat der Uebersăttigung proportional war. Etwas unsicher sind nur die Resultate beim Ammoniakalaun; dieser kristallisiert sehr viel rascher, als die anderen Stoffe. Im Anfang, etwa die ersten zwei Drittel der Uebersăttigung, sind die Konstanten gut, und dann werden sie plötzlich grőßer. Bei höherer Temperatur war dies weniger zu bemerken. Eine Erklärung fehlt noch. Im äbrigen sind die Resultate ganz eindeutig. Daß die Oberflächen sich in berechenbarer Weise durch die Kristallisation andern, konnte ich dadurch nachweisen, daß ich nach Ablauf eines Versuches die überstehende Lösung großenteils abheberte und nach Zusatz neuer ubersăttigter Lösung einen zweiten Versuch anstellte. Die Konstanten des zweiten Versuches sind genau der Berechnung entsprechend größer, als die des ersten.

Um nun weiter die Nichtanwendbarkeit der Diffusionstheorie auf die Kristallisation zu prüfen, habe ich folgende zwei Versuchsreihen angestellt. Ich habe erstens gezeigt, daß zwei verschiedene Stoffe auf derselben Oberfläche bei gleicher Uebersättigung ganz verschieden rasch kristallisieren. Zu diesem Zweck dienten mir die beiden isomorphen Salze Ammoniak - und Kalialaun.

Diese beiden Salze haben die Tugend, daß3 sie beide bei ${ }^{0}$ nahezu ganz gleich löslich sind. Ich ließ nun einmal Ammoniakalaun auf Keimen von Ammoniakalaun und dann auf Keimen von Kalialaun und ebenso einmal Kalialaun auf Kalialaun und dann auf Ammoniakalaun kristallisieren. Diese Versuche haben den Vorteil, daß die Geschwindigkeiten direkt vergleichbar werden. Es zeigte sich, daß in beiden Fällen der Ammoniakalaun I, 7 mal so rasch wächst, als der Kalialaun, und zwar konnte das in zwei Versuchsserien mit Lösungen ganz verschiedener Provenienz übereinstimmend dargetan 
werden. Das spricht meines Erachtens ganz entschieden gegen einen Diffusionsvorgang; denn es ließe sich gar kein Grund angeben, warum Ammoniakalaun nahezu doppelt so rasch diffundieren sollte, als Kalialaun.

Ferner muß, wenn die Diffusionstheorie gelten sollte, entschieden bei gleicher Oberfläche und gleichem Gefälle die Auflösung genau ebenso rasch erfolgen, wie die Abscheidung, denn der Vorgang ist ja dann nur von dem Diffusionsgefälle und der Dicke der Diffusionsschicht abhängig. Es läßt sich dieses Postulat auch mathematisch formulieren. Es wird in jedem Augenblick:

$$
V_{K}=K(E+U)-K^{\prime} E,
$$

wo $E$ die Konzentration der Sättigung, $U$ die Uebersättigung, also $E U$ die zurzeit vorhandene Konzentration bedeutet. $V_{K}$ ist die Kristallisationgeschwindigkeit. Für $U=0$ wird $K E$ $=K^{\prime} E$, woraus $K=K^{\prime}, U_{K}=K U$. Für die Auflösung gilt dann:

$$
V_{A}=K E-K(E+U)=-K U \text {. }
$$

Tatsächlich findet man nun aber, wie ich bei allen untersuchten Stoffen zeigen konnte, $\mathrm{da} B$ die Auflosung unter den gleichen Bedingungen und bei gleicher Oberfläche und gleichem Gefälle viel rascher erfolgt, als die Abscheidung. Bei dem Stoff, der am raschesten kristallisiert, dem Ammoniakalaun, ist das Verhältnis etwa $1: 4$, far den am langsamsten kristallisierenden, das Kaliumsulfat, $\mathrm{r}: \mathrm{ro}$.

Schließlich wurde auch noch der Temperaturkoeffizient untersucht. Ich konnte allerdings nur im Intervall o bis $9^{\circ}$ messen, bei höheren Temperaturen aus hier nicht năher anzufürenden Gründen nicht. Daher ist der Temperaturkoeffizient ein wenig unsicher. Ich fand far Kalialaun 1,66, Ammoniakalaun 1,44, Kaliumsulfat 1,5 und 1,6 zwischen o und ro ${ }^{0} \mathrm{C}$. Wir können also $I, 5$ bis $I, 6$ als den richtigsten Wert annehmen. Das ist für einen chemischen Vorgang nicht sehr hoch, immerhin aber wesentlich höher, als der Temperaturkoeffizient der Diffusion.

Soweit der negierende Teil meiner Arbeit, doch glaube ich auch, auf Grund meiner Resultate eine, wenn auch noch unfertige Anschauung über den Kristallisationsvorgang geben zu können.

Ich habe meine sämtlichen Messungen, deren Zahl, beiläufig gesagt, in die Hunderte geht, so berechnet, daß ich die Konzentration, die $1 / 2$ Minute nach Zusatz der Keime gefunden wurde, als Anfangspunkt meiner Messungen wablte. Dies geschah, um durch die Zeit, die zur Verteilung der Keime notwendig ist, keinen Fehler in die Rechnung hineinzubekommen; die Zeit wäde dadurch kürzer sein, als sie gemessen wurde und die Konstante zu klein gefunden werden. Als ich aber die Konstanten zwischen dem ersten und zweiten Wert doch noch berechnete, zeigte es sich zu meiner Verwunderung, daß dieselben ganz wesentlich hoher waren, als das Mittel aus den übrigen Konstanten, und zwar war das Verhältnis, je nach Stoff, Temperatur und Grad der Uebersättigung, variierend $z$ wischen $\mathrm{I}: \mathrm{x}, 8$ bis 5 . Also unter Umständen ganz enorme Differenzen. Es findet, wie hieraus klar hervorgeht, im ersten Augenblick ein anderer Vorgang statt, von dem wir zunächst feststellen können, daß er sehr rasch verläuft, denn schon nach der ersten halben Minute ist der Vorgang ganz normal geworden, und zweitens können wir feststellen, daß der primäre Vorgang in einer Stoffabgabe aus der Lösung besteht. Es ließ sich nun auch die in diesem ersten Vorgang abgegebene Menge berechnen, indem einfach mit Hilfe der gefundenen Konstanten der Anfangswert zurückberechnet wird und der so berechnete von dem tatsachlich gefundenen subtrahiert wird. Auf diese Weise ließ sich feststellen, daß die Stoffmenge, die durch diesen primären Vorgang der Lösung entzogen wird, in ganz anderer Weise von der Konzentration abhängig ist, als der sekundare Vorgang. Wahrend der letztere dem Quadrat der Uebersättigung proportional ist, ist der erstere der absoluten Konzentration angenahert proportional, wie aus der folgenden Tabelle ersichtlich ist. Dieselbe gilt für Kaliumbichromat.

\begin{tabular}{cccrr} 
Nr. & 35 & $3^{6}$ & $3^{8}$ & 39 \\
Uebersättigung . $\quad . \quad 89$ & 107 & $15^{\circ}$ & 186 \\
Konzentration . & 989 & 1007 & 1050 & 1086 \\
Primäre Verarmung & 14,5 & 14,8 & I5 & 16 \\
Hieraus berechnen & sich & \multicolumn{4}{c}{ folgende } & Verhält-
\end{tabular}
nisse:

Uebersăttigung .. . . I : I,2: $: 1,69: 2,09$

Konzentration .. . . I $: I, 02: I, 06: I, I 0$

Primäre Verarmung . . I : I,O2 : I,04: I, IO

Die Resultate sind als Differenzen ziemlich unsicher, doch durfte jedenfalls als sicher feststehen, daß die primäre Verarmung von der absoluten Konzentration und nicht von der Uebersättigung abhängig ist. Es wurde auch noch folgender Versuch gemacht: Zu Keimen, die in einer wenig gesattigten Lösung suspendiert waren, wurde neue, übersättigte Lösung gegossen. Auch hier trat, freilich in wesentlich schwăcherem Maße, primäre Verarmung ein.

Hierdurch scheint mir der Schluß ziemlich nahe gelegt, daß sich im ersten Moment um den Kristall herum eine Schicht höherer Konzentration, durch gewisse Oberflächenkrafte bedingt, herausbildet, also eine Adsorptionsschicht. Es ist so an Stelle der Noyes-Nernstschen gesattigten Schicht eine Schicht höherer Konzentration getreten. Nun kann freilich die Diffusionstheorie unter diesem Gesichtspunkte wieder aufgenommen werden, denn nun wird 
die Geschwindigkeit nicht nur von dem Konzentrationsgefalle, sondern auch von der $\mathrm{Be}$ schaffenheit des kristallisierenden Stoffes selbst abhängig.

Es ist aber wohl $z u$ beachten, daB nicht notwendigerweise die Diffusionsgeschwindigkeit der maßigebende Vorgang zu sein braucht. Es kann auch möglich sein, daß in der adsorbierten Schicht infolge bobherer Konzentration ein molekularer Vorgang stattfindet, der die Kristallisierbarkeit erst ermoglicht.

Die beiden Moglichkeiten lassen sich vielleicht so am besten präzisieren: Im ersteren Fall wird der Vorgang um so langsamer verlaufen, je grőber die adsorbierte Menge ist (groberer Diffusionsweg), im letzteren Fall um so schneller (höhere Konzentration). Die Frage steht noch offen, doch haben mir einige Versuche, die ich mit Herrn. Wenk in Jena anstellte, um diese Frage zu entscheiden, recht interessante Resultate geliefert, auf die ich nur ganz kurz eingeben will.

Wir haben den Einfluß von Zusätzen zu der Kristallisationsgeschwindigkeit untersucht und gefunden, daß für Kaliumsulfat die Geschwindigkeit durch verschiedene anorganische Stoffe erhöht oder erniedrigt wird. Um eine Beziehung zwischen diesen Beeinflussungen und der Adsorbierbarkeit der Stoffe aufzufinden, haben wir die Beeinflussung der Oberflăchenspannung flüssig-gasformig durch diese Stoffe untersucht, unter der Voraussetzung, daß hier ein Parallelismus bestehen darfe. Doch gelangten wir zu keinem Resultat, da diese Beeinflussungen zu gering sind. Wir sahen uns daher nach Stoffen um, deren Adsorbierbarkeit sich augenfällig dar- tun läßt. Als solche boten sich naturgemäB die Farbstoffe dar, von denen viele die Kristalle anfärben, andere nicht. Es zeigte sich nun, daß Farbstoffe, die nicht anfärben, auch die Geschwindigkeit nicht beeinflussen (Methylenblau), solche, die schwach anfärben, wie beispielsweise Campecheholzextrakt und Patentblau, die Geschwindigkeit deutlich verlangsamen (eine etwa 0,2 prozentige Lösung des Farbstoffes etwa auf ein Viertel der Geschwindigkeit), wăhrend stark anfärbende Farbstoffe die Kristallisation selbst dann, wenn sie in geringer Menge, 0,02 bis $0,03 \mathrm{~g}$ Farbstoff in $100 \mathrm{ccm}$, anwesend sind, fast vollständig verhindern. Bei Chinolingelb war nach 6 bis 7 Stunden noch keine meßbare Abscheidung erfolgt, obgleich wir die Uebersăttigungen zwischen 2 und $0,5 \mathrm{~g}$ bei einer Loslichkeit von $7 \mathrm{~g}$ variierten. Das gleiche Resultat ergab Ponceaurot, wăhrend bei Bismarckbraun eine eben noch bemerkbare (nach 3 Stunden mebbare) Kristallisation stattfindet. Dabei kann die adsorbierte Farbstoffmenge nicht sehr bedeutend sein, da eine wesentliche Aufhellung der Lösung nicht beobachtet wurde. Die Farbstoffzusătze haben auch auf den Habitus einen entscheidenden Einfluß, indem aus den mit Chinolingelb, Ponceaurot und Bismarckbraun gefärbten Lơsungen die Kristalle bei starker Uebersăttigung und langsamer Züchtung praktisch eindimensional wachsen, ein Zeichen dafâr, daß die Adsorption in verschiedenen Richtungen verschieden ist. Die Adsorptionstheorie gibt uns eine einwandfreie Erklärung für den Einfluß der Lơsungsgenossen auf den Kristallhabitus, eine Erklärung, die mit der Curieschen Theorie durchaus im Einklang steht. (Die Kristalle werden gezeigt.)

\section{Diskussion.}

Herr W. Biltz-Clausthal: Ich glaube, daß die interessanten Mitteilungen des Herrn Vortragenden ganz allgemein einen Beitrag zu einer Anschauung liefern, die sich doch immer mehr Geltung verschafft: als das Primäre bei jeder Reaktion.im heterogenen System eine Adsorption anzunehmen. Es kann gar kein Vorgang eintreten, wenn nicht eine molekulare Annăherung, die wir dann eben als Adsorption bezeichnen, erfolgt. Es hat sich dies in der Kolloidchemie, bei den Gasreaktionen und nun auch bei den Kristallisationserscheinungen aufs schönste gezeigt.

Ich weiß aber nicht, wie man einen Gegensatz zu der Theorie von Herrn Professor Nernst konstruieren soll; denn es geht doch evident aus seinen Arbeiten hervor, da $\beta$ es sich um eine Oberflächenschicht handelt, in der die Reaktion erfolgt; daß diese nun bei der Weiterentwicklung der Adsorptionslehre als
Adsorptionsschicht aufgefaßt wird, ist eigentlich selbstverstăndlich. Ich erinnere an das Beispiel der verschiedenen Lőslichkeit von geglühtem und frischem Eisenoxyd. Hinsichtlich der Auflösungsgeschwindigkeit scheint $\mathrm{mir}$ die Annahme einer primären Adsorptionsschicht, also einer Oberflachenwirkung, sehr klar zu sein. Für die Auskristallisation haben wir nunmehr diese, sehr wertvolle Ergänzung.

Herr W. Nernst-Berlin: Mir scheint hier doch ein prinzipielles Mißverständnis hervorzutreten. Die Theorie von Noyes, die ich später zu weiteren Schlüssen benutzt habe, besagt folgendes: wenn wir einen festen Körper in eine Lösung bringen, so stellt sich an der Oberfläche eine dünne Schicht her, in der Gleichgewicht herrscht. Vollständig davon verschieden ist die Frage des Auskristallisierens, und da darf ich vielleicht darauf aufmerksam machen, daB ich in meinem Lehrbuch der 
theoretischen Chemie die Auffassung von $\mathrm{Küster}$ adoptiert habe, daB das Auskristallisieren darauf beruht, daß nach dem Maxwellschen Leitungsgesetz eine Anzahl Konstellationen zustande kommen, so daß ein Keim sich bildet, der dann ausfällt. Daraus folgt, daß bei der Auskristallisation ein Vorgang primär mafigebend ist, der im homogenen System sich abspielt.

Herr Marc-Jena: Ich habe allerdings gerade nach der Auffassung in dem Lehrbuch ${ }^{1}$ ) des Herrn Vorsitzenden angenommen, da $B$ er die Theorie der Auflösung auf sämtliche heterogene Vorgänge ausgedehnt wissen möchte. Auch scheint mir die Annahme, daß um einen wachsenden Kristall herum stets die Konzentration der Sättigung herrscht, indem der Vorgang in der Grenzschicht sehr rasch (praktisch unendlich rasch) erfolgt, sicherlich sehr plausibel zu sein. Das Experiment hat jedoch gegen diese Annahme entschieden. Eine Steigerung der Rührgeschwindigkeit oberhalb einer gewissen Grenze ändert die Wachstumsgeschwindigkeit nicht mehr, während dies für die Auflosungsgeschwindigkeit auch nach meinen Versuchen wohl der Fall ist.

Die Kastersche Theorie, die auch von dem Herrn Vorsitzenden in seinem Lehrbuch ${ }^{2}$ ) zu der seinen gemacht worden ist, kann doch nur Anwendung auf die Bildung von Keimen finden. Hier handelt es sich aber um das Fortwachsen einer gegebenen Oberflăche. $\mathrm{DaB}$ bei meinen Versuchen neue Keime in merklicher Menge nicht gebildet worden sind und ebenso eine irgendwie erhebliche Absplitterung und damit verbundene VergroBerung der Oberflache nicht stattgefunden hat, habe ich experimentell erwiesen, indem ich einen Versuch zu Ende laufen lieb und auf den gleichen Keimen nach Abheben der Lossung einen zweiten. Die Geschwindigkeitskonstanten des Haupt- und des Folgeversuches standen dann in dem berechneten Verhältnis zueinander.

Herr M. Bodenstein-Hannover: Ich möchte den Herrn Vortragenden fragen, ob er sich eine Vorstellung aber den Grund der Gleichung der zweiten Ordnung gebildet hat.

Herr Marc-Jena: Dafür habe ich vorlaufig noch keine Erklărung, wie die Abhängigkeit von dem Quadrat der Uebersättigung zustande kommt. Der Kristallisationsprozeb wird meines Erachtens abhängig sein einmal von der Uebersăttigung und ferner von der absoluten Konzentration - dadurch wird ja die adsorbierte

I) Nernst, Theoretische Chemie, V. Auflage, S. $57^{8}$ unten.

a) Nerust, Theoretische Chemie, V. Auflage, S. 245 .
Schicht verändert. In welcher Weise aber diese adsorbierte Schicht verändert wird, ob die Dicke derselben die gleiche bleibt und die Konzentration geändert wird oder ob die Dicke sich ändert, die Konzentration aber die gleiche bleibt, oder ob beides gleichzeitig eintritt, dafür haben wir keine Anhaltspunkte, und ich meine, daß moglicherweise durch die beiden Einflusse, der Aenderung der Uebersăttigung und der Aenderung der Konzentration und der damit verbundenen Aenderung der adsorbierten Schichten ein Vorgang zuwege kommen kann, der angenähert der von mir ermittelten Gleichung entspricht. Ich habe deswegen auch vermieden, von einer zweiten Ordnung zu reden, sondern gesagt, daß der Vorgang proportional dem Quadrat der Uebersättigung erfolgt, um ihn so streng zu trennen von den Vorgängen im homogenen System.

Herr M. Bodenstein-Hannover: Ich hatte mir beim Lesen der ersten Veröffentlichung des Herrn Vortragenden vorgestellt, daß bei der Kristallisation nicht Kaliumsulfat als solches, sondern wahrscheinlich Kaliumion und Sulfation beteiligt ist, und daß möglicherweise auf dieser Tatsache eine höhere Ordnung als I beruht. Ich meine, man mäste dann auf eine Gleichung der dritten Ordnung kommen - wenn das Massenwirkungsgesetz far die starken Elektrolyte galte, und, wo das nicht der Fall ist, auf eine von ähnlicher, vielleicht genau der zweiten Ordnung. Das könnte man prüfen, wenn man die Messung bei Gegenwart überschüssigen Kalium- oder Sulfations ausfuhrt. Hat das einen Einfluß?

Herr Marc-Jena: Nein, das scheint keinen Einfluß zu haben. Ich habe Kaliumchlorid zugesetzt, ein andermal Kaliumnitrat. Kaliumchlorid beschleunigt die Reaktion, Kaliumnitrat verzogert sie um eine Kleinigkeit, sonst wird nichts geändert. Die erwähnte Annahme dürfte also wohl kaum das Richtige treffen. Dagegen hatte ich es zunăchst für möglich gehalten, daß das Zusammentreffen mehrerer Moleküle zu einem Kristallmolekül die Geschwindigkeit des Vorganges normiert. Ich habe Rechnungen ausgeführt, um dies zu prafen, doch zeigen dieselben, da $\beta$ man dann nicht zu einer Geschwindigkeit kommt, die dem Quadrat der Uebersattigung proportional ist, sondern zu einer Geschwindigkeit, die der Uebersättigung einfach proportional ist plus einem relativ kleinen $\mathrm{Zu}$ satzglied. Man gelangt also auch durch diese Annahme allein nicht zu der von mir gefundenen Beziehung. Fur die habe ich demnach vorlăufig keine Erklärung. Ich glaube, wie gesagt, daß der Vorgang komplexer Natur ist. 